\title{
A PHYSICS BASED DEEP LEARNING TECHNIQUE FOR PROGNOSTICS
}

\author{
Khaled Akkad \\ Department of Mechanical and Industrial Engineering \\ University of Illinois at Chicago \\ kakkad2@uic.edu
}

\begin{abstract}
Remaining useful life (RUL) estimation is one of the most important aspects of prognostics and health management (PHM). Various deep learning (DL) based techniques have been developed and applied for the purposes of RUL estimation. One limitation of DL is the lack of physical interpretations as they are purely data driven models. Another limitation is the need for an exceedingly large amount of data to arrive at an acceptable pattern recognition performance for the purposes of RUL estimation. This research is aimed to overcome these limitations by developing physics based DL techniques for RUL prediction and validate the method with real run-to-failure datasets. The contribution of the research relies on creating hybrid DL based techniques as well as combining physics based approaches with DL techniques for effective RUL prediction.
\end{abstract}

\section{PROBLEM STATEMENT}

Prognostics focus on predicting the time to failure of industrial systems and components. Time to failure is often referred to as the RUL. Prognostics are important for condition based maintenance as it provides accurate timelines for maintenance scheduling and component replacement. In recent years, DL has received much attention for developing data-driven prognostic solutions. In the age of Internet of Things (IoT) and big data, due to the availability and widespread development of sensing technology, massive amounts of data are continuously collected for condition monitoring.

The availability of data has driven researchers and companies to pursue prognostics using DL approaches. The ability of DL approaches to capture the underlying intrinsic patterns of massive data is one of the main reasons for its success in prognostic applications. DL

Khaled Akkad et al. This is an open-access article distributed under the terms of the Creative Commons Attribution 3.0 United States License, which permits unrestricted use, distribution, and reproduction in any medium, provided the original author and source are credited. based methods have shown great scalability and robustness when used with high dimensional datasets. DL based prognostics are less prone to produce effective models when used with low dimensional datasets.

In order to further improve the accuracy of RUL estimation and overcome the limitations of DL, physics based approaches will be combined with DL based approaches. While DL techniques can capture the underlying patterns in highly nonlinear systems, it is expected to perform even better when applied on linear systems. The Koopman operator [1] as a physics based approach is one of the promising tools to combine with DL based approaches. The Koopman operator is a linear transformation tool that obtains infinite linear representations of dynamical systems. One of the most promising techniques to approximate Koopman modes is the dynamic mode decomposition (DMD).

For the purposes of RUL estimation, DL based approaches can be applied as the main prognostic technique after the data had been preprocessed by the Koopman operator. This approach would be considered a hybrid method that combined both physics based and DL based approaches sequentially. Figure 1 shows the potential sequential flow of this method with convolutional neural networks (CNN) and long short term memory (LSTM) networks as the main DL based approach, as an example.

\section{EXPECTED CONTRIBUTIONS}

The implementation of this research includes the following potential contributions:

(1) The development of a new hybrid prognostic method that incorporates physics based modeling with DL based approaches.

(2) The validation of the physics based DL technique using experimental run-to-failure data of turbofan engines, gears, and bearings.

\section{RESEARCH PLAN}

3.1. The development of physics based DL technique for prognostics 


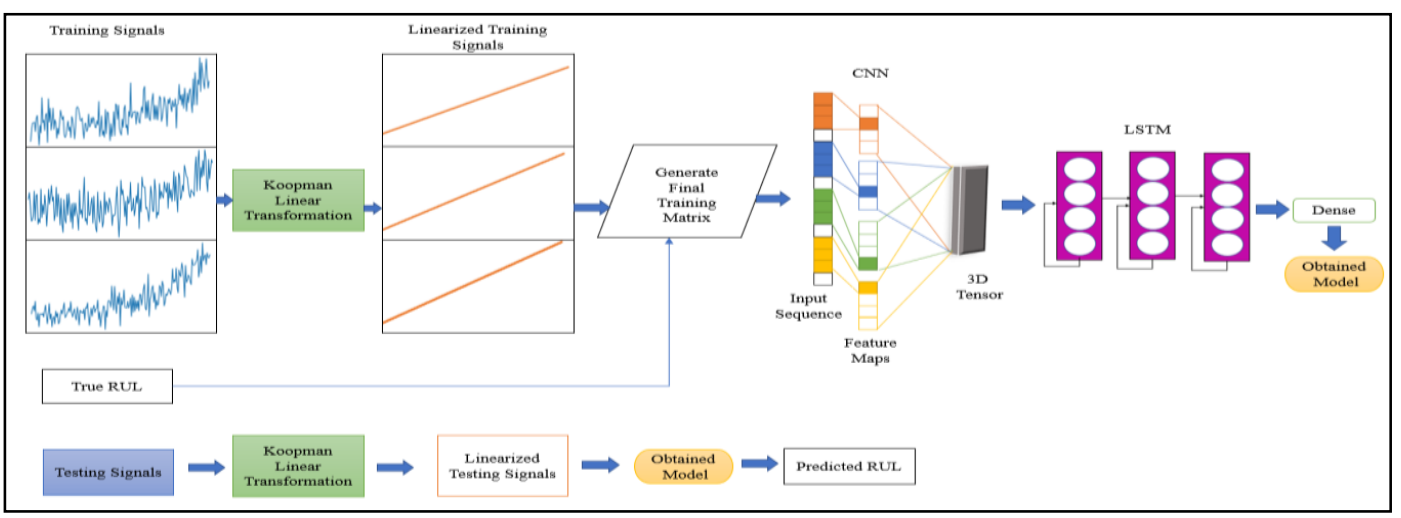

Figure 1. Koopman-LSTM-CNN flow

The first step is to incorporate the Koopman linear transformations into different DL structures. Different preprocessing procedures will then be applied on the linearized signals to allow for a smooth input into the first layer of the deep neural network depending on the specific network type. The second step is to use a hybrid DL based approach to process the obtained linearized signals. Meta-learning is to be used to derive the best combination of DL based approaches. The third step is to estimate the RUL of a benchmark dataset to test the validity of the proposed method.

\subsection{Test the physics based DL approach using three datasets}

To validate the proposed method, different datasets will be used for the purposes of RUL estimation. The first dataset will be the NASA C-MAPSS turbofan engines datasets. Figure 2 shows the flow of air in a simulation engine in the C-MAPSS dataset. The second dataset will be a bevel gear dataset as shown in Figure 3. Finally, bearing data will be used for validating the proposed method. The test rig for the bearing data is shown in Figure 4.

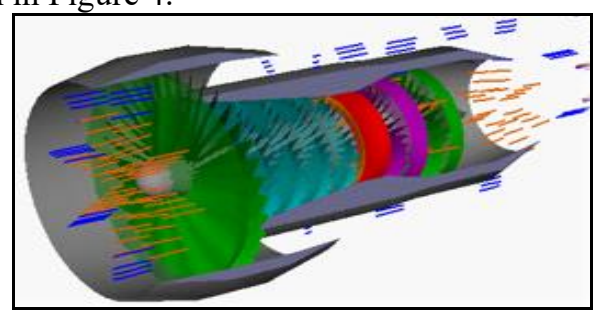

Figure 2. Airflow through C-MAPSS engine

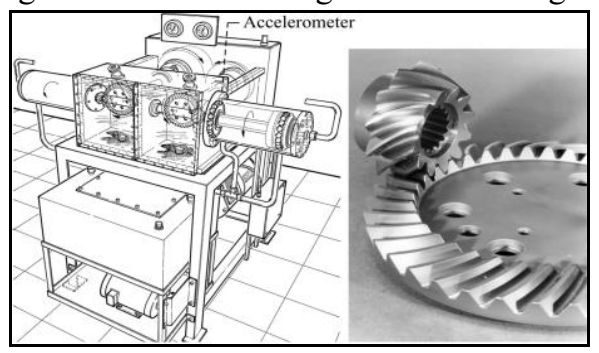

Figure 3. Bevel gear test rig and bevel gears [2]

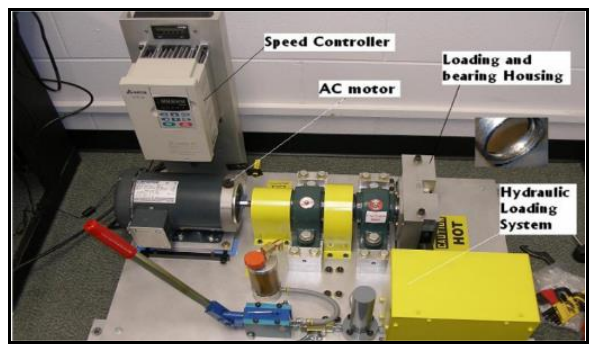

Figure 4. Bearing run-to-failure test rig

\subsection{Performance Assessment}

The last step in the research plan is to perform some assessment tasks to ensure the effectiveness as well as the efficiency of the proposed method. Assessment tasks include: (1) calculate the runtime for each of the deep hybrid combinations as well as the Koopman mode extraction which will help when scaling the method to much larger datasets, (2) analyze the loss of information resulted from the Koopman transformation of signals, (3) RMSE of the predicted RUL.

\subsection{Work Performed}

A hybrid DL based model was developed to estimate the RUL of the C-MAPSS engines. One-dimensional CNN and LSTM networks were sequentially combined to create the hybrid model. Figure 5 shows the basic structure of the used method. The CNN portion of the structure is inspired by [3]. Table 1 shows both the training and testing trajectories in the utilized datasets for validating the performed hybrid method on the CMAPSS data.

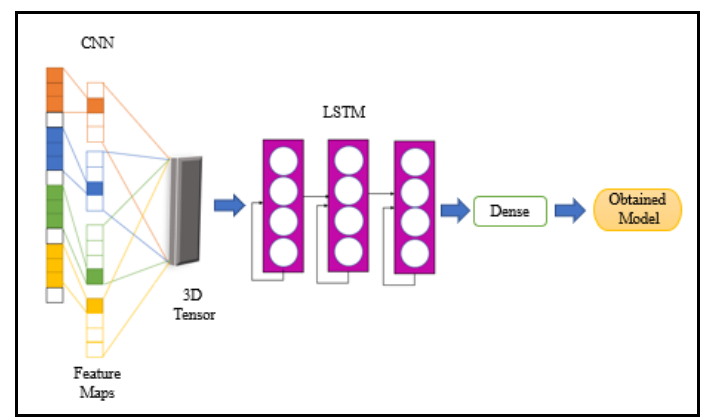

Figure 5. Hybrid deep learning structure 
Table 1. C-MAPSS dataset

\begin{tabular}{|c|c|c|c|}
\hline Dataset & \multicolumn{3}{|c|}{ Engine Fleet } \\
\hline & FD001 & FD002 & FD003 \\
\hline Train Trajectories & 100 & 260 & 100 \\
\hline Test Trajectories & 100 & 259 & 100 \\
\hline
\end{tabular}

When validating the hybrid DL based model, it was compared to a pure LSTM model to demonstrate the effectiveness of the hybrid model. It was found that the hybrid model outperforms the pure LSTM model. Figures 6 and 7 show the validation loss and RUL estimation comparison, respectively, of the pure LSTM against the hybrid model. It is worth noting that only one of the 3 C-MAPSS datasets used is shown in terms of both validation loss and RUL comparison due to limited space. After that, Table 2 shows the average RMSE values of the predicted RUL.

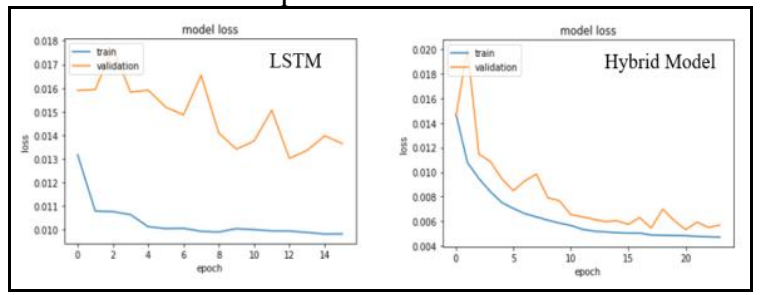

Figure 6. Validation loss for FD002

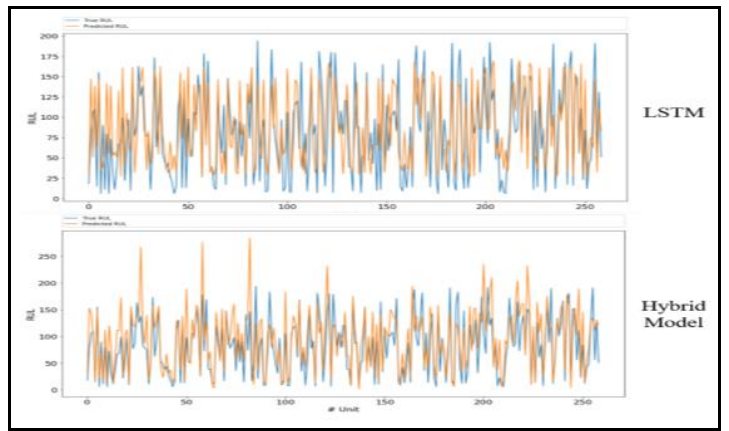

Figure 7. True RUL (Blue) vs. predicted RUL (Orange)

Table 2. RMSE Values

\begin{tabular}{|c|c|c|c|c|}
\hline Method & FD001 & FD002 & FD003 & Average \\
\hline LSTM & 27.77 & 38.49 & 31.09 & 32.45 \\
\hline Hybrid Model & 22.13 & 36.35 & 23.79 & 27.42 \\
\hline
\end{tabular}

For this research, DMD was applied on vibration signals to obtain linear representations of the data that are easier to process for RUL estimation. It was found the Koopman modes compresses the vibration signal to an almost constant function. It seems like the Koopman operator can be used for more complicated systems than ones whose sensor readings are timeseries vibration signals. For that reason, it is intended to further explore how the Koopman operator can be used efficiently for simpler systems. If successful, the representations obtained by the Koopman operator could produce perfectly linear modes that can be used for RUL estimation. Since linear processing tools are well developed, it is expected to arrive at much better prognostic models when the data is preprocessed with the Koopman operator. Figure 8 shows the signal after compression.

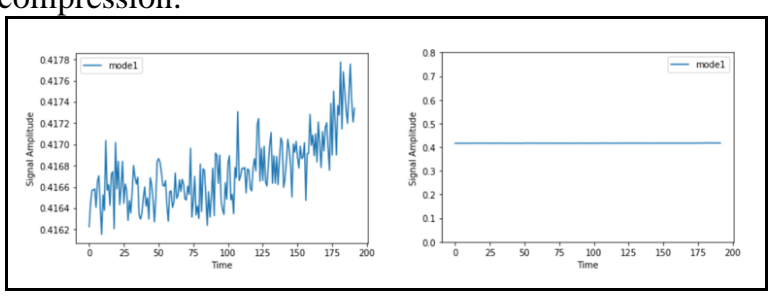

Figure 8. Koopman mode of vibrations signals

\subsection{Remaining Work}

The next steps in this research are as follows:

(1) Replace the sequential model in Figure 1 with one that utilizes Autoencoder (AE) to reconstruct vibration signals with respect to a target that is the output of the Koopman linearization.

(2) Integrate the Koopman decomposition into different DL structures including LSTM, CNN, and AE.

(3) Use meta-learning to optimize the combination of DL based hybrids.

(4) Develop a Koopman-DL model for prognostics and validate the model on the mentioned datasets.

\section{CONCLUSION}

The development of new prognostic method is crucial to further improving RUL estimation. A hybrid DL based prognostic approach was tested for RUL estimation. The results show that the hybrid method outperforms the pure LSTM method. It is the intent of this research to overcome the limitations of DL. The logical next step is to find more suitable combinations of DL based approaches using meta-learning. After that, this suitable combination will be used to process data that has been modified using a physics based approach called Koopman decomposition. The output of this potential model is expected to produce more accurate RUL estimation.

\section{REFERENCES}

[1] Koopman, B. O., "Hamiltonian systems and transformations in Hilbert space", Proceedings of the National Academy of Sciences, vol. 17, no. 5, pp. 315318, 1931.

[2] Dempsey, P., Handschuh, R., and Afjeh, A., "Spiral bevel gear damage detection using decision fusion analysis", in Proceedings of the Fifth International conference on Information Fusion, Annapolis, MD, USA, 2002.

[3] Li, X., Ding, Q., and Sun, J-Q., "Remaining useful life estimation in prognostics using deep convolution neural networks," Reliability Engineering and System Safety, vol. 172, pp. 1-11, 2018. 